\title{
PERFIL MULTIDIMENSIONAL DE LA EMPATÍA EN ESTUDIANTES DE LA LICENCIATURA EN CRIMINOLOGÍA DE LA UNIVERSIDAD AUTÓNOMA DE NUEVO LEÓN.
}

\section{MULTIDIMENSIONAL PROFILE OF EMPATHY IN STUDENTS OF THE DEGREE IN CRIMINOLOGY OF THE AUTONOMOUS UNIVERSITY OF NUEVO LEÓN}

\author{
Benjamin Salvador Simón, Reyna L. Vázquez Gutiérrez \\ Universidad Autónoma de Nuevo León \\ Correspondencia: benjaminsalvadorsimon@hotmail.com
}

\section{RESUMEN}

El concepto de la empatía y su relación con las profesiones en las que se brinda un servicio a un usuario en crisis ha sido ampliamente estudiado. A este respecto la criminología, es una de ellas, puesto que trabaja con personas que han estado implicadas en algún delito, por lo que se ha de tomar en consideración, además de la preparación académica, las aptitudes y las actitudes de las personas que proveerán el servicio directo. Se debe entonces establecer un perfil claro al respecto de la empatía, basada en el modelo multidimensional de ésta. Por ello el objetivo del presente artículo es evaluar el perfil multidimensional de empatía que presentan los y las estudiantes de la licenciatura en criminología, de la Universidad Autónoma de Nuevo León (UANL). La muestra del estudio se conformó con las y los estudiantes de los diferentes semestres incluidos en el turno piloto de la Facultad de Derecho y Criminología UANL ( $=162$ ), se aplicó el Test de Empatía Cognitiva y Afectiva (TECA). Se concluyó que el perfil multidimensional de la empatía de los y las estudiantes de criminología, se caracteriza por puntuaciones significativamente más bajas a las deseables, cuestión que nos hace reflexionar sobre la inclusión de contenidos de corte aptitudinal en los programas de formación integral, incidiendo en la formación y el desarrollo personal como un elemento que garantice el adecuado desempeño profesional futuro. 
Palabras Clave: Empatía; Criminología; Estudiantes; Empatía Cognitiva; Empatía Afectiva.

\section{ABSTRACT}

The concept of empathy and its relationship with the professions in which a service is provided to a user in crisis has been extensively studied. In this regard, criminology cannot be separated from these professions, since it works with people who have been involved in some crime, either as offenders, as well as primary or secondary victims, so it must be taken into consideration, in addition to the academic preparation, skills and attitudes of the people who will provide the direct service. A clear profile should then be established regarding empathy, based on its multidimensional model, to which the people currently conducting criminology studies have to adjust, not only with respect to theoretical content, let alone judge their abilities based on them, but it is also convenient to take into consideration other relevant aspects that may mediate in the professional performance of their profession, thus guaranteeing a quality and human service such as that which concerns us. Therefore, the objective of this article is to evaluate the multidimensional profile of empathy presented by criminology students, in order to know if they have adequate skills for their professional performance at the time of graduation. The sample with which they have worked are the students of the different semesters included in the pilot shift of the Faculty of Law and Criminology UANL ( $N=162$ ), through the application of the Cognitive and Affective Empathy Test (TECA), and the subsequent analysis of results using the SPSS software. Finally, a reflection is made on the possible professional consequences that can be derived from the results found after the students leave.

Key words: Empathy; Criminology; Students; Cognitive Empathy; Affective Empathy.

\section{INTRODUCCIÓN}

La importancia de que las personas profesionales de la criminología posean unos adecuados niveles de empatía es crucial para su desempeño profesional, puesto que es el elemento principal que mediará en su sus relaciones con las personas que ocupan de sus servicios, puesto que éstas han vivido situaciones vitales difíciles como víctima o victimario, o han tenido una pérdida importante a raíz 
de esas situaciones, por lo que una actuación basada en la empatía, entre otras aptitudes y actitudes marcará la diferencia. No obstante, tal y como se verá a continuación, dentro de la empatía pueden encontrarse varios componentes, por lo que se hace necesario una evaluación multidimensional, y por consiguiente deberemos de comenzar desarrollando una revisión bibliográfica con respecto al constructo para proporcionar posteriormente una definición de éste acorde a la perspectiva propuesta.

Ahora bien, más allá de su importancia con respecto al campo de la criminología, el constructo de la empatía se constituye como una variable fundamental cuando queremos comprender la conducta humana, encontrándose que se encuentra muy relacionada con el comportamiento prosocial (Coke, Batson y McDavis, 1978). Cabe destacar que nos encontramos ante un elemento complejo, el cual puede ser interpretado, junto con su desarrollo, de formas diferentes atendiendo a las cosmovisiones teóricas, o a los enfoques o corrientes científicas bajo los que hayan sido postuladas dichas interpretaciones, lo que a su vez también guía la investigación hacia diferentes líneas para contrastar teorías alternativas e incluso contrapuestas. A pesar de que parece existir cierto consenso sobre la expresión conductual de la empatía, sigue sin alcanzarse un pleno acuerdo sobre su definición a nivel global (Fernández-Pinto, López-Pérez y Márquez, 2008).

Para comprender nuestro tema de estudio, se ha de partir de una revisión de la bibliografía de mayor relevancia con respecto al constructo de la empatía, tratando de desglosar sus efectos sobre las variables secundarias, para exponer finalmente el abordaje conceptual que se adoptará en la presente tesis con el fin de realizar una aclaración del constructo.

\section{Concepto de empatía.}

Etimológicamente, empatía proviene del término griego Empháteia (sentir afecto dentro, o sentirse dentro de), pero se instaura a finales del Siglo XVIII en base al germanismo Einfühlung (intropatía) acuñado por Robert Vischer (citado en Davis, 1996) y que podría traducirse por "sentirse dentro de". Comenzaba a dejar ver la concepción actual que tenemos sobre la empatía, es decir, ponerse en los zapatos del otro, o entrar en su piel para comprender y experienciar sus vivencias, no 
obstante, hasta 1909, no se acuñó el término empatía, momento en el que Titchener (citado en Davis, 1996) vuelve a retomar la etimología griega previamente citada, $\epsilon \mu \varpi \dot{\alpha} \theta \epsilon \iota \alpha$.

Las definiciones más clarificadoras del constructo comienzan a aparecer en el siglo XX, y los autores Fernández-Pinto, López-Pérez y Márquez (2008) realizan una síntesis de la evolución histórica de las definiciones más relevantes, síntesis que tomaremos como base de este apartado, encontrando que: Lipps (citado en Wispé, 1986) considera que la empatía se desarrolla mediante un proceso de imitación interna, mediante el que las personas nos proyectamos en los demás, y por consiguiente entendemos su situación como si la estuviésemos atravesando nosotros. En la primera mitad del siglo XX comienza a aparecer también la diferenciación entre los enfoques cognitivo y afectivo de la empatía. Köhler (citado en Davis, 1996) es pionero del enfoque cognitivo y comprende la empatía como la capacidad del ser humano para comprender los sentimientos ajenos. Mead (1934) sigue esta perspectiva, y añade la adopción de perspectivas como necesaria para comprender los sentimientos de los otros. En la otra mano, la perspectiva afectiva comienza en 1947, con Fenichel (citado en Davis, 1996), quien entiende la empatía como la capacidad de identificación con el otro, base de diferentes teorías posteriores en las que se encuentra una perspectiva social o situacional (Igartua, y Páez, 1998), entendiendo como un acontecimiento importante el desarrollo y la posterior introducción del término adopción de perspectivas o roletaking (Dymond, 1959). Posteriormente Hogan (1969) tomaría estas aportaciones para definir la empatía como el proceso mental por el que construimos los estados mentales subjetivos, o lo que es lo mismo, la forma en la que comprendemos y compartimos lo que ocurre en la mente de otros, instaurándose así como una capacidad meta-representativa. La perspectiva de Hogan ha encontrado evidencia científica que parece apoyarla (ver: Sharmay-Tsoory, Tormer, Goldsher, Berger y Aharon-Peretz, 2004; Elliot et al., 2006). En psicología podemos comprender que el acontecimiento más relevante en el estudio de la empatía fue cuando en 1951, Carl Rogers hiciese énfasis en que se trataba de un concepto de esencial importancia para el éxito de cualquier intervención terapéutica.

El primer punto que debemos de abordar a la hora de definir la empatía, es el de acotar el constructo, puesto que existen diferentes conceptos que parecen similares al de empatía, como simpatía, altruismo o compasión. Estos conceptos 
realmente no alcanzan el nivel de precisión que debe de tenerse al hablar de la empatía, puesto que ha de comprenderse desde los planos cognitivo y afectivo/ emocional. La empatía es entendida por la mayor parte de la población como la capacidad que nos permite o nos facilita percibir y entender los sentimientos de los demás, así como sus comportamientos y/o su motivación, de manera que actúe como una vacuna frente al surgimiento de ciertos tipos de conflictos. A pesar de que esta característica parecería propiamente inherente al ser humano, hay individuos que presentan unos niveles de empatía casi inexistentes, derivándose en configuraciones patológicas de la personalidad y/o propensión hacia los delitos y las conductas antisociales.

\section{Componentes de la empatía.}

Históricamente, en el estudio de la empatía podemos encontrar que unos autores se centran el rasgo emocional/conductual (empatía afectiva), entendida como la capacidad de experimentar y compartir las emociones de otros (Mehrabian \& Epstein, 1972) y actuar en consecuencia, mientras su vez, otros autores se focalizan en el rasgo cognitivo (empatía cognitiva), que ha sido descrito como la capacidad para comprender las emociones de los demás (Hogan, 1969) (Fernández-Pinto, López-Pérez y Márquez; 2008). Otros autores toman la empatía como un conjunto de ambos rasgos (Cohen \& Strayer, 1996). Ahora bien, tomando una definición más actual (Ang \& Goh, 2010), la empatía se entiende como un constructo multidimensional que está compuesto por un rasgo cognitivo y un rasgo afectivo (emocional/conductual). A este respecto, nos encontramos ante uno de los principales problemas con relación al constructo, es decir, saber si debemos comprender la empatía definida como la capacidad de "ponerse mentalmente en el lugar del otro", o si hemos de ir más allá y ver que se refiere a la capacidad de "sentir la emoción de forma vicaria" (Chlopan, McCain, Carbonell y Hagen, 1985), por "experiencia vicaria" debemos comprender todas aquellas emociones y todos los sentimientos que se vivencien a través de las situaciones vividas por los demás.

A pesar de no encontrarse un consenso científico sobre la definición exacta de ambos tipos de empatía, sí que se ha alcanzado dicho consenso con respecto a su 
existencia y su componente diferencial, si bien este pequeño nivel de acuerdo con respecto sus componentes diferenciales, no termina de eliminar las controversias que rodean al constructo que nos atañe en el presente trabajo.

\section{Empatía Cognitiva.}

Podría decirse que, los investigadores que se adscriben en esta línea de comprensión de la empatía, la ven como un proceso intelectual de toma de perspectivas, aún con las variaciones en sus definiciones o caracterización sobre la empatía. Los acercamientos académicos al estudio de la empatía que la comprenden desde una perspectiva cognitiva, la definen como la capacidad intelectual que permite a los individuos ver y comprender el mundo desde la perspectiva de los otros (Duan y Hill, 1996; Gladstein, 1983), aunque existen otras perspectivas sobre la empatía cognitiva donde se comprende como la habilidad de sentir o entender los estados mentales, las experiencias o los sentimientos de los demás sin perder la perspectiva objetiva del observador (Hogan, 1969; Kohut, 1959; Rogers, 1957).

A través de procesos imaginativos guiados por instrucciones, Stotland (1969) encuentra que las personas somos capaces de sentir ansiedad cuando nos imaginamos a nosotros mismos en una situación, pero que no somos capaces de sentir esa ansiedad cuando nos imaginamos a otra persona en esa situación. Davis (1996), siguiendo la línea de Stotland, encontró que aquellos sujetos que obtenían mayores puntuaciones en adopción de perspectivas, tendrían unas mayores puntuaciones también con respecto a la capacidad de imaginarse a los demás. Se ha encontrado una relación inversamente proporcional entre el componente cognitivo de la empatía, o lo que es lo mismo, la adopción de perspectivas, y la agresividad (Richardson y Malloy, 1994), entendiendo así que las personas más violentas y/o agresivas mostrarían un déficit en su capacidad para comprender o imaginarse a los otros, y por consiguiente no podrían adoptar su postura y comprender el daño que pueden causarles.

La perspectiva cognitiva de la empatía, comprendida principalmente como la capacidad de la adopción de la perspectivas mental del otro (cognitive role-taking), muestra relativa similitud con el constructo de teoría de la mente, que se define como la habilidad que poseemos los seres humanos para dar explicaciones sobre el comportamiento propio o de los otros, así como para anticiparlo, al atribuirle 
a dicha conducta una carga cognitiva, o lo que es lo mismo, unas necesidades, intereses, intenciones, y emociones, entre otros procesos cognitivos.

\section{Empatía afectiva.}

En la otra mano, diferentes autores han adoptado una perspectiva afectiva de la empatía, la cual consiste en la comprensión de la empatía como las reacciones emocionales que se producen ante las expresiones emocionales de los demás (e.g., Gladstein, 1983; Hoffman, 2000). Los estudios de Stotland (1969), a pesar de encontrarse recogidos en el apartado cognitivo por su carga respecto a la adopción de perspectivas, fueron lo primeros en comprender a la empatía como un afecto que se comparte en función de la emoción del otro, o como un sentimiento vicario. Stotland define la empatía como "la reacción emocional de un observador que percibe que otra persona está experimentando o va a experimentar una emoción" (1969, p.272). Hoffman (1987), en cambio, comprende la empatía como la respuesta afectiva que los seres humanos generamos ante la situación de otra persona. Mehrabian y Epstein (1972) continúan la línea propuesta por Hoffman, y definen la empatía como la respuesta afectiva vicaria ante las vivencias emocionales de los otros, o lo que es lo mismo la emoción o el sentimiento compartido, sentir lo que el otro siente, siendo de esta forma una disposición condicionada por las diferencias individuales. Wispé (1978), se centró en destacar la importancia de los estados emocionales positivos dentro del concepto de empatía, puesto que hasta ese momento siempre se había relacionado con los sentimientos de compasión ante las desgracias ajenas.

El elemento común entre los autores previamente citados es que consideran la empatía como una disposición individual, pero se encuentra también una perspectiva situacional de la empatía afectiva, siendo de especial relevancia la aportación de Batson (1991), que entiende la empatía como el resultado de tomar conciencia sobre el malestar de los otros, generando así un sentimiento vicario congruente con su estado afectivo. Por consiguiente la empatía, es comprendida como una respuesta elicitada ante un estímulo emocional externo (Fernández-Pinto, LópezPérez y Márquez, 2008), siendo importantes los trabajos experimentales de Coke, Batson y McDavir (1978), de los que deviene la creación de un inventario para medir esta respuesta elicitada. 


\section{El enfoque multidimensional de la empatía, la perspectiva integradora.}

Comprendemos que las aproximaciones cognitivas y/o afectivas de la empatía se encuentran muy limitadas, y han sido duramente criticadas puesto que suponen un reduccionismo de las capacidades empáticas de los sujetos. Diferentes autores postulan que los componentes de la empatía pueden ocurrir en conjunto o incluso uno como consecuencia del otro, instaurándose así como componentes primario y/o secundarios (Clark, 2007; Kerem, Fishman, y Josselson, 2001). Los enfoques o aproximaciones multidimensionales sobre el constructo de la empatía, nos ayudan a comprender la complejidad del mismo, dado que lo entienden como un entramado de múltiples procesos que pueden ocurrir exclusiva o conjuntamente. Una de las primeras aproximaciones multidimensionales es la de Davis (1983), quien postulaba que la empatía se encuentra compuesta por las reacciones de la persona ante las experiencias observadas de otras personas, bien sean cognitivas o afectivas. Para defender su tesis, crea el instrumento Índice de Reactividad Interpersonal (Interpersonal Reactivity Index, IRI; Davis, 1983) donde define la empatía en base a 4 subescalas comprendidas, a su vez, en dos escalas superiores que corresponden a las dimensiones cognitiva (Toma de Perspectivas; y Fantasía) y afectiva (Preocupación Empática; y Aflicción Personal) de la empatía.

Tal y como se ha podido observar, los estudios diferenciales sobre el contenido cognitivo y afectivo de la empatía llega hasta la década de los 80, momento en el que se comienza a trabajar en aportar definiciones integradoras de la empatía, - lo que es lo mismo, se adopta la perspectiva multidimensional a partir de los trabajos de Davis (1980) en los que realiza la primera definición multidimensional y la propuesta que culminaría en el inventario previamente referenciado. Pero no fue hasta que Eisenberg y Strayer (1987) estableciesen la distinción entre la adopción de perspectiva perceptual, cognitiva, y afectiva, cuando cristalizó esta visión integradora, al incluir la adopción de la perspectiva perceptual, comprendida como la capacidad de comprensión sobre lo que la otra persona puede percibir en base a la localización física de ésta. No obstante, se han desarrollado diferentes estudios que tratan de poner a prueba la hipotesis de la independencia entre lo componentes de la empatía, encontrando una falta de congruencia en los resultados, puesto que unos sí apoyaban la existencia de esa independencia (Mill, 1984; Smither, 1977; Gladstein, 1983), mientras que otros la refutaban (Hoffman, 1984). 
El Modelo Organizacional (Davis, 1996) pretende realizar un análisis de la empatía aludiendo a sus antecedentes, sus procesos y sus consecuentes. Una de las críticas al modelo, indicada por el propio autor (Davis, 1996), es que se indica que los elementos se encuentran relacionados de manera unidireccional, imposibilitando así la interrelación de los elementos de una forma más compleja, y a su vez tampoco se ha tenido en cuenta las características de la persona sobre la que se va a empatizar, lo que no parece estar indicando cuándo van a aparecer unas respuestas u otras (Fernández-Pinto, López-Pérez, y Márquez, 2008). El autor nos ha dejado a su vez la definición de empatía más aceptada en la actualidad, entendiéndola como "conjunto de constructos que incluyen los procesos de ponerse en el lugar del otro y respuestas afectivas y no afectivas" (Davis, 1996, p.12).

Es importante indicar que, desde los años 90, la empatía se ha incluido en la perspectiva de la inteligencia emocional (término acuñado por Salovey y Mayer, 1990), pero se ha de mencionar que esta inclusión limita el constructo de la empatía, al contemplarlo como una capacidad o habilidad cognitiva, desatendiendo su componente afectivo (Salovey y Mayer, 1990).

Llegados a este punto, y tras haber conceptualizado el constructo central de nuestra investigación en base a una revisión bibliográfica extensa, se desprende como objetivo de la presente investigación el contrastar la validez del instrumento TECA (Test de Empatía Cognitiva y Afectiva; López-Pérez, Fernández-Pinto y Abad; 2008) con las y los estudiantes de criminología de la Facultad de Derecho y Criminología de la Universidad Autónoma de Nuevo León para establecer posteriormente un perfil promedio de los niveles de empatía que presentan.

\section{MÉTODO.}

Objetivo General: Evaluar el perfil multidimensional de empatía que presentan los y las estudiantes de la Licenciatura en Criminología de la Universidad Autónoma de Nuevo León.

Participantes: La selección de las personas participantes en el presente estudio se ha llevado a cabo mediante una selección aleatorizada de las aulas donde se aplicó el instrumento, durante el turno piloto de impartición de la licenciatura en criminología en la Facultad de Derecho y Criminología de la Universidad Autónoma 
de Nuevo León, tomando en consideración un balance entre los diversos semestres que se cursan en ese horario. El número de muestra resultante ha sido de 162 individuos participantes, que se dividen en 65 hombres (40.1\% de la muestra) y 97 mujeres (59.9\% de la muestra), presentando una edad media de 19.07 años, con una desviación típica de 1.674, siendo el mínimo y el máximo de edad 17 y 27 respectivamente.

Instrumento: El instrumento que se ha utilizado en este estudio nace de la definición de empatía que se utilizará será la propuesta por Davis (1996) donde queda descrita como "conjunto de constructos que incluyen los procesos de ponerse en el lugar del otro, y de respuestas afectivas y no afectivas", siendo recogida por López-Pérez, Fernández-Pinto y Abad (2008; p.6) en el manual de su instrumento TECA (Test de Empatía Cognitiva y Afectiva), instrumento que se ha elegido para aplicar en el apartado cuantitativo, y mantiene una perspectiva multidimensional de la empatía.

El motivo por el que se ha elegido este cuestionario (TECA), y por consiguiente la definición de la empatía que le subyace, es que incorpora una escala destinada a evaluar las emociones y sentimientos positivos, aspecto que resulta novedoso frente a otros instrumentos como es el caso de IRI (Índice de Reactividad Interpersonal, Davis, 1980). A su vez adopta una perspectiva multidimensional al incorporar el componente cognitivo frente a otro de los instrumentos con mayor relevancia, pero que solamente evalúa el componente afectivo de la empatía, el QMME (Cuestionario de Empatía Emocional, Mehrabian y Epstein, 1972).

El instrumento TECA (López-Pérez, Fernández-Pinto y Abad, 2008) se basa en los dos elementos principales de la empatía previamente explicitados, el cognitivo y el afectivo, pero a su vez propone una división de cada uno en dos escalas, las cuales serían:

\section{-Componente cognitivo:}

a) Adopción de perspectivas (8 ítems)- Esta escala proviene del término anglosajón cognitive role taking (i.e. Eisenberg y Strayer, 1987; Davis, 1996), y hace referencia al conjunto de procesos cognitivos de carácter intelectivo y/o imaginativo que nos permiten situarnos a nosotros mismos en el lugar de otro. 
b) Comprensión empática (9 ítems)- Esta escala hace referencia a la capacidad que tenemos para identificar y entender el aparato cognitivo, volitivo y afectivo de los demás, o lo que es lo mismo, comprender sus emociones, intenciones e impresiones. A pesar de que se incluye un elemento del componente afectivo, esta escala es puramente cognitiva puesto que no exige de compartir el sentimiento o la emoción, solamente exige de su comprensión a nivel cognitivo.

\section{-Componente afectivo:}

c) Estrés empático (8 ítems)- Esta escala refiere la capacidad individual para compartir las emociones negativas que los otros presentan, la capacidad de acomodarnos a su sentir a este respecto.

d) Alegría empática (8 ítems)- Esta escala incluye la capacidad de cada persona para compartir las emociones positivas de los otros. Es la versión positiva de la escala previa, y por consiguiente son complementarias.

El instrumento es de aplicación rápida, entre 5 y 10 minutos y puede aplicarse de manera individual o colectiva y, además, solamente necesita de una escolarización básica para su comprensión y se compone de un total de 33 ítems divididos entre las subescalas de la forma previamente indicada, mostrando adecuadas propiedades psicométricas, con una fiabilidad general de a .86, y una validez de criterio de $r .63$ con respecto al QMEE (Questionaire Measure of Emotional Empathy; Mehrabian y Epstein, 1972), y de r .73 con respecto al IRI (Interpersonal Reactivity Index; Davis, 1980), tal y como se indican en el manual del instrumento (López-Pérez, FernándezPinto y Abad, 2008).

\section{RESULTADOS}

\section{Resultados generales.}

Los datos han sido codificados y analizados con el software estadístico IBM SPSS Statistics en su versión 25. Tras codificar los datos y realizar las inversiones en las puntuaciones de los ítems, tal y como se refiere en el manual, se procede a realizar un análisis de la fiabilidad del instrumento a través del estadístico Alfa de Cronbach, desglosando las puntuaciones tal y como se indica en el manual, e incorporando 
la medida de fiabilidad para los componentes cognitivo y afectivo de la empatía, obteniendo los resultados que se pueden observar en la Tabla 1.

\begin{tabular}{lcc}
\multicolumn{1}{|c}{ Reporte de fiabilidad (alfa de Cronbach) por escala } & a Estudio & a Manual \\
\hline Puntuación global (33 ítems) & .805 & .86 \\
1. Empatía cognitiva (17 ítems) & .761 & XX* \\
\hline 1.1. Adopción de Perspectivas (8 ítems) & .685 & .7 \\
1.2. Comprensión Empática (9 ítems) & .645 & .74 \\
\hline 2. Empatía Afectiva (16 ítems) & .709 & $\mathrm{XX}^{*}$ \\
2.1. Estrés Empático & .624 & .78 \\
2.2. Alegría empática & .762 & .75
\end{tabular}

Tabla 1. Comparación entre las puntuaciones de fiabilidad (a de Cronbach) en la aplicación del instrumento en el presente estudio, frente a la fiabilidad mostrada en el manual original. $\mathrm{XX}^{*}$ Indica que las puntuaciones de fiabilidad para el conjunto de ítems incorporados en el factor no se muestra en el manual original del instrumento.

Mediante la utilización del método de análisis de fiabilidad de las dos mitades o Slipt Half, se demuestra la estructura bifactorial del instrumento, o lo que es lo mismo, la presencia de los componentes cognitivo y afectivo, encontrando además una correlación de Spearman-Brown de .581 entre los dos factores, cuestión que nos indica que ambos factores están relacionados dentro del constructo, pero que no evalúan lo mismo, es decir, que son puntuaciones de dos componentes diferenciados dentro del mismo constructo.

Como se ha podido ver, el instrumento muestra unas adecuadas propiedades con respecto a la fiabilidad en la muestra en la que ha sido aplicado, pero cuando realizamos la prueba de normalidad de Kolmogorov-Smirnov (prueba adecuada puesto que nuestra muestra es mayor a 50 sujetos) obtenemos un estadístico de .085 con una significación de .007 lo que nos indica que los datos obtenidos en nuestra muestra no presentan una distribución normal.

\section{Perfil multidimensional de empatía en la muestra.}


En primer lugar, se ha de hacer constar que no se encuentran diferencias estadísticamente significativas en base a las variables sociodemográficas, o lo que es lo mismo en base al sexo y/o a la edad. En la Tabla 2. se presentan de manera sintética los resultados promedio que se observa en la muestra de estudiantes de criminología sobre la que se ha aplicado el instrumento.

\begin{tabular}{lcccccc}
\multicolumn{1}{c}{ Escala } & $\begin{array}{c}\text { Puntuación } \\
\text { máxima }\end{array}$ & $\begin{array}{c}\text { Media } \\
\text { Observada }\end{array}$ & $\begin{array}{c}\text { Mínimo } \\
\text { observado }\end{array}$ & $\begin{array}{c}\text { Máximo } \\
\text { observado }\end{array}$ & $\begin{array}{c}\text { Puntuación } \\
\text { centil* }\end{array}$ & Significación* \\
$\begin{array}{l}\text { Comprensión } \\
\text { Emocional }\end{array}$ & 45 & 32,25 & 19 & 45 & 65 & Media \\
$\begin{array}{l}\text { Adopción de } \\
\text { Perspectivas }\end{array}$ & 40 & 29,13 & 14 & 39 & 50 & Media \\
\hline $\begin{array}{l}\text { Alegría Empática } \\
\text { Estrés Empático }\end{array}$ & 40 & 33,60 & 9 & 40 & 70 & Alta \\
\hline $\begin{array}{l}\text { Componente Cognitivo } \\
\text { Componente Afectivo }\end{array}$ & 85 & 21,52 & 11 & 34 & 30 & Baja \\
\hline Puntuación Total & $\mathbf{6 0}$ & $\mathbf{6 1 , 3 8}$ & 36 & 82 & $\mathrm{XX}$ & $\mathrm{XX}$ \\
\hline
\end{tabular}

Tabla 2. Resultados de la aplicación del instrumento en nuestra muestra. Se puede observar por orden la puntuación máxima que puede ser alcanzada, seguido de la puntuación promedio observada en la muestra, así como las puntuaciones mínimas y máximas observadas en la muestra para cada escala. *La puntuación centil y la significación se extraen directamente del manual del instrumento. ${ }^{* *}$ En el caso de las puntuaciones de Componente Cognitivo y Componente Afectivo no se presentan puntuaciones centiles ni significación en el manual.

-Comprensión Empática: El promedio observado en nuestra muestra nos arroja una significación media con respecto a esta escala. Esto nos indica que la muestra, a nivel general, no parece ser capaz de hacer una lectura adecuada de las emociones con respecto a su expresión verbal, no verbal y paraverbal, cuestión que podría incidir en sus relaciones con las personas usuarias, especialmente con respecto a las entrevistas, recogida de información y comunicación de avances en las investigaciones.

-Adopción de Perspectivas: En esta escala ocurre lo mismo que en la anterior, la significación es media, lo que nos dice que parece existir un pensamiento inadecuadamente flexible por defecto, cuestión que pudiera interferir en la comunicación y en la interacción con otras personas, al no ser capaces de adecuar sus formas de pensar a los diferentes contextos que puedan presentarse en su desempeño profesional en la misma línea que la escala anterior. 
-Componente Cognitivo: A pesar de que en el manual del instrumento (LópezPérez, Fernández-Pinto y Abad, 2008) no se incluye una interpretación del componente cognitivo en su conjunto, podemos interpretar, en base a las dos escalas que lo componen, una significación media. A este respecto, la muestra no parece presentar un pensamiento flexible que le permita tomar en consideración las posiciones de otras personas y por consiguiente actuarán de una manera rígida y acorde a sus propias posiciones, cuestión que limitaría su desempeño, especialmente en el trato directo cara a cara, así como en la toma de declaraciones, entrevistas y recogida de información.

-Alegría Empática: La muestra presenta una puntuación en esta escala de la que se desprende una significación alta, punto que nos indica que las personas que componen la muestra, por término medio, exhiben una facilidad a la hora de compartir los estados emocionales positivos de otras personas, cuestión que facilita sus interacciones con las personas, fortaleciendo sus redes sociales. A este respecto, la puntuación en la escala incidirá en el desempeño profesional de los y las profesionales de la criminología en la medida en que mediante sus respuestas ante las emociones de los demás pueden motivar a las personas para mantener los cambios que éstas presenten, y que sean positivos para el futuro de las mismas.

-Estrés Empático: Con respecto a la escala inversa a la anterior, la muestra presenta una significación baja. Esta puntuación se relaciona con la facilidad con la que las personas empatan emocionalmente con las emociones negativas de los otros. Al tratarse de una puntuación baja, encontramos que nuestra muestra, de manera general, se caracteriza por una frialdad emocional en esta línea, premiando sus propias necesidades y emociones ante las de las otras personas, sin importar cómo les hagan sentir. Con respecto al desempeño profesional de la criminología, esta indiferencia hacia las víctimas, ofensores, y/o familiares de ambos, puede incidir en una mala atención que genere una revictimización o una mala praxis en el desempeño de sus funciones.

-Componente Afectivo: Al igual que ocurre con respecto al Componente Cognitivo, en el manual del instrumento (López-Pérez, Fernández-Pinto y Abad, 2008) tampoco se toma este factor o conjunto de escalas, pero en base a las escalas que los componen podemos extraer una significación media. Estos resultados, tomando en consideración las diferencias entre las escalas, nos indican que los 
y las estudiantes de criminología comparten las emociones positivas de otras personas, mientras que pueden permanecer indiferentes ante las emociones negativas, cuestión que puede interferir con su desempeño profesional al trabajar especialmente con personas que han sufrido una situación vital negativa, y por consiguiente sus emociones se presentarán en esta línea.

-Puntuación total: En este punto, solo queda indicar que la muestra manifiesta una puntuación global que puede traducirse a una significación media, lo cual parece indicar que los niveles globales de empatía no son óptimos para un desarrollo personal y profesional adecuado.

En el siguiente apartado se discutirán los resultados encontrados, observando la adecuación de los mismos al perfil deseable de las personas profesionales de la criminología tras su egreso.

\section{CONCLUSIONES}

Se debe comenzar este apartado mencionando que los resultados de la aplicación del instrumento indican unas adecuadas propiedades psicométricas, al encontrarse en nuestra muestra un buen nivel de fiabilidad, muy similares a las puntuaciones a de Cronbach que se recogen en el manual del instrumento. No obstante, encontramos varias limitaciones en el presente estudio que inciden en el hecho de que no se puedan generalizar los resultados de la investigación, siendo la principal el que no se presenta una distribución normal en nuestra muestra, pero a su vez se ha dispuesto de una muestra pequeña, que si bien es representativa del turno en el que las mediaciones se han llevado a cabo, puede tener diversas particularidades que actúen como variables moderadoras, por lo que se haría necesaria la replicación de este estudio incluyendo una muestra más amplia y que recoja alumnas y alumnos de los diferentes turnos de manera balanceada.

Ahora bien, con respecto al perfil personal y profesional deseable en el desempeño de la criminología con respecto a la empatía, podríamos incidir en la necesidad de la presencia de puntuaciones que muestren una significación alta en todas las escalas, cuestión que, como se ha visto en el apartado anterior, solo se cumple con respecto a la escala Alegría Empática. Ahora bien, no se deberían de presentar puntuaciones con una significación extremadamente alta, puesto que su desempeño profesional se vería influenciado en demasía por las personas usuarias, 
fomentando a su vez el fenómeno de burnout, a través del impacto emocional de las tragedias ajenas sobre la persona. El perfil deseado de los y las profesionales de la criminología con respecto al componente cognitivo sería aquel caracterizado por una comunicación eficiente basada en la tolerancia y en la flexibilidad cognitiva, que además de permitirle comprender las situaciones que se le presente, le permita también comprender la posición de los demás, a través de una interpretación eficiente de su comportamiento y su lenguaje (verbal, no verbal y paraverbal), que le facilite la interpretación, a nivel cognitivo de los estados emocionales de las otras personas. Con respecto al componente cognitivo entendemos que el perfil deseado de los criminólogos y las criminólogas sería aquel caracterizado por una adecuada comprensión, y adecuación de las conductas a los estados emocionales, tanto positivos como negativos, de las otras personas. En este punto es importante incidir en que si bien es necesario una puntuación alta en este componente, se ha de dotar de elementos, herramientas y estrategias a los estudiantes para que sean capaces de tomar cierta distancia emocional hacia las situaciones vitales de los otros para no incurrir en un sesgo dentro de sus actuaciones hacia la parcialidad, y a su vez generando una huella psicológica en los y las profesionales de la criminología.

Tal y como se han observado en los resultados del presente artículo, el perfil multidimensional de la empatía de los y las estudiantes de criminología que componen nuestra muestra, se caracteriza por unas puntuaciones significativamente más bajas a las deseables, cuestión que nos hace reflexionar sobre la inclusión de contenidos de corte aptitudinal en los programas de formación integral de las diferentes universidades, incidiendo en la formación y el desarrollo personal como un elemento que garantice el adecuado desempeño profesional futuro, puesto que los conocimientos no determinan acciones por si mismos, sino que han de acompañarse por diferentes habilidades o disposiciones personales que garanticen un adecuado cumplimiento con las funciones y que a su vez el servicio provisto posea la adecuada calidad humana, acorde a los derechos humanos de las personas que soliciten el servicio.

Como bien se ha dicho, la muestra sobre la que se ha realizado el presente análisis no muestra una distribución normal, y a su vez podríamos estar incurriendo en una restricción en el rango de los resultados debida a las características de la propia muestra, por lo que debemos incidir en la necesidad de una mayor cantidad de investigación y/o evidencia científica que nos permitan establecer la relación 
entre un perfil generalizable de los y las estudiantes de criminología, tanto a nivel estatal como a nivel nacional con respecto al perfil deseable arriba mencionado. En esta línea, el presente trabajo podría servir como punta de lanza para otras investigaciones futuras que nos permitan establecer la inclusión de la empatía, así como otras capacidades, habilidades y/o actitudes que incidan directamente en el desempeño profesional de los criminólogos con respecto a los planes de formación integral propuestos por las casas de estudios, a fin de preparar a las y los estudiantes de criminología de una manera holística.

\section{REFERENCIAS}

I Ang, R. P., y Goh, D. H. (2010). Cyberbullying among adolescents: The role of affective and cognitive empathy, and gender. Child Psychiatry y Human Development, 41, 387-397.

I Batson C. D. (1991) The altruism question: Toward a social psychological answer. Hillsdale: Lawrence Erlbaum.

I Carlo, G., Mestre, M. V., McGinley, M. M., Samper, P., Tur, A., y Sandman, D. (2012). The interplay of emotional inestability, empathy, and coping on prosocial and aggressive behaviors. Personality and Individual Differences, 3, 675-680.

I Chlopan, B. E., McCain, M. L., Carbonell, J. L., y Hagen, R. L. (1985) Empathy: review of available measures. Journal of personality and social psychology, 48, 635-653

I Clark, M. A. (2007). Cognitive and affective empathy: Exploring the differential effects of empathy components on work-family conflict and emotional labor (Tesis de maestría no publicada). Disponible en: ProQuest Dissertations and Theses database. (UMI No. 1446883).

I Cohen, D., y Strayer, J. (1996). Empathy in conduct-disordered and comparison youth. Developmental Psychology, 32, 988-998.

I Coke, J., Batson, C., y McDavis, K. (1978) Empathetic mediation of helping: A two-stage model. Journal of Personality and Social Psychology, 36, 752-766.

I Davis, M. H. (1980) A multidimensional approach to individual differences in empathy. Catalog of Selected Documents in Psychology, 10, 1-17. 
I Davis, M. H. (1983). Meassuring individual differences in empathy: Evidence for a multidimensional approach. Journal of personality and social psychology, 44, 113-126.

I Davis, M. H. (1996). A Social Psychological Approach. Boulder: Westview Press.

I Decety, J., y Yoder, K. J. (2016). Empathy and motivation for justice: Cognitive empathy and concern, but not emotional empathy, predict sensitivity to injustice for others. Social Neuroscience, 11, 1-14.

I Duan, C., y Hill, C. E. (1996). The current state of empathy research. Journal of Counseling Psychology, 43, 261-274

I Dymond, R. R. (1949). A scale for the measurement of empathic ability. Journal of Consulting Psychology. 13, 127-133.

I Eisenberg, N., y Fabes, R. A. (1992). Emotion, regulation, and the development of social competence. En M. S. Clark, Emotion and social behavior (pp. 119-150). Thousand Oaks: Sage.

I Eisenberg, N., Fabes, R. A., y Spinrad, T. L. (2006). Prosocial development. . En N. Eisenberg, W. Damon, y R. M. Lerner, Handbook of child psychology Vol 3: Social, emotional and personality development (6th ed.) (pp. 646-718). Hoboken: Wiley.

I Eisenberg, N., y Strayer, J. (1987) Empathy and its development. Nueva York: Cambridge University Press.

I Elliott, R., Völlm, B., Drury, A., McKie, S., Richardson, P., y Deakin, J. F. W. (2006). Cooperation with another player in a financially rewarded guessing game activates regions implicated in theory of mind. Social Neuroscience, Special Issue: Theory of Mind, 1, 385395.

I Fernández-Pinto, I., López-Pérez, B., y Márquez, M. (2008) Empatía: Medidas, teorías y aplicaciones en revisión. Anales de Psicología, 24, 284-298.

I Frick, P. J., Cornell, A. H., Bodin, S. D., Dane, H. B., Barry, C. T., y Loney, B. R. (2003). Callous-unemotional traits and developmental pathways to severe conduct problems. Developmental Psychology, 39, 246-260.

I Gladstein, G. A. (1983). Understanding empathy: Integrating counseling, developmental, and social psychology perspectives. Journal of Counseling Psychology, 30, 467-482. 
I Hoffman, M. L. (1984a) Interaction of affect and cognition in empathy. In C. E. Izard, J. Kagan y R.B. Zajonc (Eds.) Emotions, cognition and behavior, pp.103-131. Nueva York: Cambridge University Press.

1 Hoffman, M. L. (1987). The contribution of empathy to justice and moral judgment. En N. Eisenberg y J. Strayer, Empathy and its development (pp. 47-80). Nueva York: Cambridge University Press.

1 Hoffman, M. L. (2000). Empathy and moral developement: Implications for caring and justice. Nueva York: Cambridge University Press.

I Hogan, R. (1969). Development of an empathy scale. Journal of Consulting and Clinical Psychology, 33, 307-316.

I Igartua, J. J., y Páez, R. D. (1998) Validez y Fiabilidad de una escala de empatía e identificación con los personajes. Psicothema 10, 423-436.

I Kerem, E., Fishman, N., Josselson, R. (2001). The experience of Empathy in everyday relationships: Cognitive and affective elements. Journal of Social and Personal Relationships, 18, 709-729.

I Kohut, H. (1959). Introspection, Empathy, and Psychoanalysis-An Examination Relationship Between Mode of Observation and Theory. Journal of American Psychoanalytic Association, 7, 459-483.

ILópez-Pérez, B., Fernández-Pinto, I., y Abad, F. J. (2008). Manual del Test de Empatía Cognitiva y Afectiva TECA. Madrid: TEA Ediciones.

I Mead, M. (1934). Mind, self, and society. Chicago: University of Chicago Press.

I Mehrabian, A., y Epstein, N. (1972). A measure of emotional empathy. Journal of Personality, 40, 525-543.

I Mill, J. (1984). High and low self-monitoring individuals: Their decoding skills and empathic expression. Journal of Personality, 52, 372 - 388.

I Richardson, E. D., y Malloy, P. F. (1994) The frontal lobes and content especific decisions. Journal of neuropsychiatry and clinical neurosci-ence, 6, 455-466.

I Rogers, C. R. (1951). Client-Centered Therapy: Its Current Practice, Implications, and Theory, Boston: Houghton Mifflin. 
$\checkmark$ Rogers, C. R. (1957). The Necessary and Sufficient Conditions of Therapeutic Personality Change. Journal of Consulting Psychology, 21, 95-103.

I Salovey, P. y Mayer, J.D. (1990). Emotional intelligence. Imagination, Cognition and Personality, 9, 185-211.

I Sharmay-Tsoory, S.G., Tormer, R., Goldsher, D., Berger, B. D., y Aharon-Peretz, J. (2004) Impairment in Cognitive and Affective Empathy in Pa-tients with Brain Lesions: Anatomical and Cognitive Correlates. Journal of Clinical and Experimental Neuropsychology, 26, 11131127.

I Smither, S. (1977). A reconsideration of the developmental study of empa-thy. Human development, 20, $253-276$.

I Stotland, E. (1969). Exploratory investigations of empathy. En Berkowitz (Ed.) Advances in experimental social psychology, 4, p.272.

I Wispé, L. (1986) The distinction between sympathy and empathy: To call for a concept a word is needed. Journal of Personality and Social Psychology, 50, 314-321.

Envío a dictamen: 13 de noviembre de 2019

Reenvío: 11 de diciembre de 2019 Aprobación: 18 de diciembre de 2019 